\title{
The Non-homogeneous Groshev Convergence theorem for Diophantine Approximation on Manifolds
}

\author{
Faiza Akram, Dongsheng Liu \\ Department of Mathematics, School of Science, Nanjing University of Science and \\ Technology,Nanjing, Jiangsu, 210097, P.R. China \\ Faiza.akram786@yahoo.co.uk
}

\section{ABSTRACT}

This paper is based on Khintchine theorem, Groshev theorem and measure and dimension theorems for nondegenerate manifolds. The inhomogeneous Diophantine approximation of Groshev type on manifolds is studied. Major work is to discuss the inhomogeneous convergent theory of Diophantine approximation restricted to non-degenerate manifold $M$ in $R^{n}$, based on the proof of Barker-Sprindzuk conjecture, the homogeneous theory of Diophantine approximation and inhomogeneous Groshev type theory for Diophantine approximation, by the decomposition of the set in manifold, with the aid of Borel Cantell lemma and transformation of $(C, \alpha)-$ good lemma and its properties and the main inhomogeneous conversion principle, we know these two types of set in sense of Lebesgue measure is zero provided that the convergent sum condition is satisfied, from which several conclusions about the inhomogeneous convergent theory of Diophantine approximation is obtained. The main result is that Lebesgue measure is inhomogeneous strongly extremal. At last we use the fact that friendly measure is strongly contracting measure to develop an inhomogeneous strong extreme measure which is restricted to matrices with dependent quantities.

\section{Keywords}

Inhomogeneous transformation principle, Inhomogeneous Diophantine approximation, Groshev type theorem, Inhomogeneous strongly extremal

\section{INTRODUCTION}

In number theory, the idea of rational approach was long ago, People often use a rational approximation and approximation to represent a certain number, the development and on the basis of Diophantine approximation (Diophantine Approximation) is a historical theory a long and important branch, For example, we are familiar with the history of Pie, is the rational approximation of irrational numbers $\pi$, the real relatively systematic approximation theory was developed in 19th Century with the establishment of the theory of real numbers, Now it has become one of the most active branches of number theory.

The development of Diophantine approximation theory can be broadly divided into two major categories, Respectively, The Diophantine approximation in primitive number theory and the Diophantine approximation on Manifolds, Among them, the former research history is relatively long, the main research questions are: (1) Approximation of a single real number; (2) Simultaneous approximation of multiple real numbers; (3) Homogeneous approximation and nonhomogeneous approximation; (4 ) Rational approximation of algebraic numbers; ( 5 ) Uniform distribution of Diophantine approximation; ( 6 ) Metric theorem; ( 7 ) Uniform sequence distribution; ( 8 ) $\boldsymbol{\rho}$ - adic Diophantine approximation, etc, See literature [1] for details. These are the classical results of linear Diophantine approximation. The second kind is developed in recent years and new branch of approximation theory, and has become a more active part. Especially, the approximation of the vector or matrix of parametric variables with the method of power system is a good result. From 1932, K.Mahler proposed the Veronese curve $M=\left\{\left(x_{1} x^{2}, \cdots, x^{n}\right): \in R\right\}$ since almost all points are not the beginning of very well approximable's conjecture, the Diophantine approximation of manifolds has become an active research topic, Its main research questions are: (1) Metric Diophantine approximation on manifolds embedded in Homogeneous Spaces; (2) Metric Diophantine approximation on manifolds embedded in non-homogeneous spaces; (3) Metric, Diophantine, approximation, etc. of Khitchine - Groshev type on Manifolds; Manifold Diophantine approximation is a very important and very wide application areas, $\mathrm{n}$ has important academic significance and application value for the study, researchers from different angles to analyze, put forward many methods and techniques of Diophantine approximation, and establishes a relatively perfect Diophantine approximation theory.

This paper mainly studies the manifold dual Diophantine approximation non-homogeneous theory problem, namely homogeneous dual Diophantine approximation of Khintchine - Groshev theorem to the non-homogeneous dual Diophantine approximation. By classifying the points on the set $A_{n}^{P}(\emptyset) \cap M$, The transformation from nonhomogeneous to homogeneous is applied by using the kernel of deformation, $(C, \alpha)-$ good function and its properties and the nonhomogeneous transformation principle in the literature. Given in the series and convergence under the condition of limited in $A_{n}^{p}(\emptyset) \cap M$ on the measure theory, that is the collection of the set $A_{n}^{p}(\emptyset) \cap M$ has Lebesgue measure 0 , In order to launch a non homogeneous strong extreme, this excellent generalized metric Diophantine approximation theory on manifolds Khitchine - type Groshev. Also with the help of friendly measure is a strong contraction in fact at the end of this paper, further promotion of a class of non homogeneous strongly extreme measure with variable matrix space. 


\section{Preliminary knowledge}

\subsection{Definition of Diophantine approximation and its symbolic representation}

Start with the definition of the most general matrix Diophantine approximation:

Definition 1.1: For any $Y \in M_{m_{1} n}$ there is $\delta>0$ such that for infinitely many $q \in Z^{n}$ and the corresponding $p \in Z^{m}$ there is $|Y q-p|<|q|^{-n / m-\delta}$ holds. Then $\mathrm{Y}$ is called very well approximable (Abbreviation VWA).

Here $|X|=\max \left|x_{i}\right|$ is the maximum norm.

Definition 1.2: For any $Y \in M_{m, n}$ there is $\delta^{\prime}>0$ such that for infinitely many $q \in Z^{n}$ and the corresponding $p \in Z^{m}$ there is $\Pi(Y q-p)<\Pi_{+}(q)^{-\left(1+\delta^{\prime}\right)}$ holds. Then $\mathrm{Y}$ is called very well approximable (Abbreviation VWMA).

Here $\Pi(X) \stackrel{\text { daff }}{=} \prod_{i}\left|x_{i}\right|$ and $\prod_{+}(X) \stackrel{\text { deff }}{=} \prod_{i} \max \left(\left|x_{i}\right|, 1\right)$.

From the definition we known that $q \in Z^{n}$ and $q \neq 0$ there are $\Pi(Y q-p) \leq|Y q-p|^{m}$ and $\Pi_{+}(q) \leq|q|^{n}$, then there is

$$
\prod(Y q-p) \leq|\mathrm{Yq}-\mathrm{p}|^{\mathrm{m}}<\left(|\mathrm{q}|^{-\mathrm{n} / \mathrm{m}^{-\delta}}\right)^{\mathrm{m}}=|\mathrm{q}|^{-\mathrm{n}\left(1+\frac{\mathrm{m}}{\mathrm{n}} \delta\right)} \leq \prod_{+}(\mathrm{q})^{-\left(1+\frac{\mathrm{m}}{\mathrm{n}} \delta\right)}
$$

so here $\delta^{\prime}=\frac{\mathrm{m}}{\mathrm{n}}-\delta$. When $Y \in M_{m_{n} n}$ is VWA then its means that $Y$ is also VWMA.

If the measure is under $\mu$, for almost all $Y \in M_{m, n}$ is not VWA, then $\mu$ is called extreme. Similarly, in the measure $\mu$, for almost all $Y \in M_{m_{2} n}$ is not VWMA, then $\mu$ is called strongly extreme. The set of VWA matrices is zero under the Lebesgue measure, but the dimension of the set is the dimension of the $M_{m_{n} n}$ under the Hausdroff measure.

On the non-homogeneous Diophantine approximation, defined as follows:

Definition 1.3: Let $Y \in M_{m, n}, z \in R^{m},(Y, z)$ is called VWA, if there is $\delta>0$ for infinite $q \in Z^{n}$ with the existence of the corresponding $p \in Z^{m}$ and $|Y q+z-p|<|q|^{-\frac{n}{m}-\delta}$ holds. Similarly, by using the definition $Y q+z$ can be replace the above $Y q$, is called VWMA.

\section{Definition1.4:}

$A_{n}^{P}(\Phi):=\left\{x \in R^{n}\right.$ : there are infinitely many $q \in Z^{n} \backslash\{0\}$ such that $\left.\|q * x+P(x)\|<\Phi(q)\right\}$, when $x \in A_{n}^{P}(\Phi)$ it is called $(\Phi, P)$ approximation.

Where $\|x\|=\inf f|x-p|: p \in Z\}$. $\Phi: R^{n} \rightarrow \mathrm{R}^{+}$is an $n$ approximation function, which satisfy $\left|q_{i}\right| \leq\left|\mathrm{p}_{\mathrm{i}}\right|$, when there are $i=1,2, \cdots, n$ available.

$$
\Phi\left(q_{1}, \cdots, q_{n}\right) \geq \Phi\left(p_{1}, \cdots, p_{n}\right)
$$

In particular, we can make $\Phi(q)=\phi(\mid \mathrm{ql})$ here $\phi: R^{+} \rightarrow \mathrm{R}^{+}$is an approximation function.

By definition, if $\Phi(\alpha)=\phi(|\mathrm{q}|)$, remember that $A_{n}^{P}(\Phi)=\mathrm{A}_{\mathrm{n}}^{\mathrm{P}}(\phi)$ holds.

When $P=$ Constant, it is equivalent to the dual Diophantine approximation under non-homogeneous conditions. When $P=0$, it is equivalent to the dual Diophantine approximation under homogeneous conditions, then it is denoted as: $A_{n}(\Phi)$.

Definition 1.5: If $\mu \in U, f$ in $\mu$ as the center of the main ball full length $l$ continuous differentiable, and $f$ in the $\mu$ point of the $l$ order partial derivative can be made into $R^{n}$, it is said that $f$ is $l$-non-degenerate at $\mu \in U$. 
If $f$ in $U$ then almost all points are $l$-non-degenerated, $f$ is called $l$-non-degenerate. If there exist $l$ such that $f$ is $l$-nondegenerate, then $f$ is said to be non degenerate. While the definition of manifold non-degeneration is: if $M$ is embedded in the $m$ dimensional $R^{n}$ manifolds, there is a non-degenerate mapping $f: U \rightarrow R^{n}$, (Here $U \in R^{m}$ ) there is $M:=\mathrm{f}(\mathrm{U}) \cdot M$ is said to be non-degenerate.

Lemma 1.1: (Reference [6] Borel-Cantelli Lemma) suppose that $(\Omega, A, P)$ is a probability space, and $\left\{A_{i}\right\}$ is some column probability event, then:

a: if $\sum_{n=1}^{\infty} P\left(A_{n}\right)<\infty$, then $P\left(\right.$ limsup $\left.A_{n}\right)=0$;

b: If the event $A_{n}$ is an independent event, and $\sum_{n=1}^{\infty} P\left(A_{n}\right)=\infty$, then $P\left(\limsup A_{n}\right)=1$.

It is easy to apply lemma to Lebesgue measure: when $\sum_{q \in Z^{n} \backslash\{0\}} \Phi(q)<\infty$, there is a corresponding $|A(\Phi)|=0$.

Let $m$ tuples $\beta=\left(\beta_{1}, \beta_{2}, \cdots, \beta_{m}\right), \beta_{i}$ is the nonnegative integer, and so, $|\beta|_{8}:=\beta_{1}+\cdots+\beta_{\mathrm{m}}$ $\partial_{\beta}:=\frac{\partial^{\mid \mathrm{p}]_{*}}}{\partial x_{m}^{\beta_{1}} \cdots \partial x_{m}^{\beta_{m}}}$ and $\alpha_{i}^{k}:=\frac{\partial^{k}}{\partial x_{i}^{k}}$.

Lemma 1.2: (Reference [2] Lemma 1.3) let $U$ be an open subset of $R^{m}, g \in \mathrm{C}^{k}$ (U) makes the existence of constants $A_{1}, \mathrm{~A}_{2}>0$, there is $\left|\partial_{\beta} g(x)\right| \leq \mathrm{A}_{1}, \forall \beta, \quad|\beta|_{*} \leq \mathrm{k}$ for all $x \in U$, and $\left|\partial_{i}^{k} g(x)\right| \geq \mathrm{A}_{2}$, $\forall i=1, \cdots, m$ let $U$ is a subset of $V$ and arbitrary extreme cube $V$ in ball $B$ are contained in $U$, then $g$ is $\left(\mathrm{C} \frac{1}{m k}\right)$. good on $V$.

The constant $C$ depends only on $A_{1}, \mathrm{~A}_{2}, \mathrm{~m}, \mathrm{k}$.

Here the Vinogradov symbols $\ll$ and $\gg$ indicate that the multiplicative constant can be eliminated for an inequality, if $a \ll b$ and $a \gg b$ it can be written as $a=b, B \equiv B(x, r)$ means that $x \in R^{m}$ is also a ball with radius of $r$, then $\lambda B(x, r):=\mathrm{B}(\mathrm{x}, \lambda \mathrm{r})$.

\section{2 non-homogeneous transformation principle}

This section introduces the main content of the document [2], that is, the non-homogeneous transformation principle, here we combine the specific application, let $V$ express $R^{m}$ as a open ball, $\mu$ is a $m$ dimensional Lebesgue measure that is restricted to $V$, the support set of $U$ on $V$ is the closure $\bar{V}$ and remember: $S:=\bar{V}$.

Let $T$ and $A$ be two countable index sets, let $\mathrm{H}$ and $\mathrm{I}$ are all open subset of mapping $T \times A \times R^{+}$to $R^{m}$, that is:

$$
H:(t, \alpha, \varepsilon) \mapsto H_{f}(\alpha, \varepsilon) \text { and } I:(t, \alpha, \varepsilon) \mapsto I_{f}(\alpha, \varepsilon)
$$

Let $\Psi$ denote the set of all $\psi: T \rightarrow R^{+}$functions, if $\psi \in \Psi$ consider the supremum of the set.

$$
\Lambda_{I}(\psi):=\lim _{t \in T} \sup \bigcup_{\alpha \in A} I_{t}(\alpha, \psi(t)) \text { and } \Lambda_{H}(\psi):=\lim _{t \in T} \sup \bigcup_{\alpha \in A}(\alpha, \psi(t))
$$

The next two properties are used to ensure the supremum set on the "homogeneous" $\Lambda_{H}(\psi)$ is transformed into the supremum set of "non-homogeneous" $\Lambda_{I}(\psi)$. 
Intersection property: If for any $\psi \in \Psi$, the presence of $\psi^{*} \in \Psi$ makes it possible to do so except for a limited number of $t$, for all $t \in T$ and different $\alpha, \alpha^{1} \in A$, and

$$
I_{t}(\alpha, \psi(t)) \cap I_{t}(\alpha, \psi(t)) \subset \bigcup_{\alpha \in A} H_{t}\left(\alpha^{\mathrm{m}}, \psi^{*}(t)\right)
$$

It is called the three tuple $(H, I, \psi)$ intersection property.

Contraction property: If any $\psi \in \Psi$ and a series of positive numbers $\left\{k_{t}\right\}_{t \in T}$ are allowed

$$
\sum_{t \in T} k_{t}<\infty
$$

In addition to a limited number of $t$, for all $t \in T$ and all $\alpha \in A$, there is a set $\mathrm{S}$ of the ball $\mathrm{B}$ in the center $C_{t, \alpha}$, satisfies the following three conditions:

$$
\begin{aligned}
& s \bigcap_{t}(\alpha, \psi(t)) \subset \bigcup_{B \in C_{t, \alpha}} B \\
& s \bigcap \bigcap_{B \in c_{t, \alpha}} B \subset I_{t}\left(\alpha, \psi^{*}(t)\right)
\end{aligned}
$$

and

$$
\mu\left(5 B \cap I_{t}(\alpha, \psi(t))\right) \leq \mathrm{k}_{\mathrm{t}} \cdot \mu(5 \mathrm{~B})
$$

Theorem 1.1: (Reference [1], Theorem 3)

Assumptions $(H, I, \Psi)$ satisfy intersection properties, $\mu$ is about $(I, \Psi)$ contradiction, then

$$
\forall \psi \in \Psi_{,} \mu\left(\Lambda_{H}(\psi)\right)=0 \Rightarrow \forall \psi \in \Psi_{,} \mu\left(\Lambda_{I}(\psi)\right)=0 .
$$

\section{$2(C, \alpha)-$ good function and its qualitative}

Definition 2.1: (Reference [4]) Let $C$ and $\alpha$ be positive numbers, $f: V \rightarrow R$ is a function that defines the set $V$ on $R^{m}$. If there is a arbitrary open ball $B \subset V$ and $\forall \varepsilon>0$ there is

$$
\mu\left(\left\{x \in B: f|(x)|<\varepsilon \cdot \sup _{x \in B}|f(x)|\right\}\right) \leq \mathrm{C} \varepsilon^{\alpha} \mu(\mathrm{B})
$$

then $f$ is $(C, \alpha)-$ good on $V$.

The $(C, \alpha)-$ good function has the following properties.

\section{Lemma 2.1:}

(a) If $f$ is $(C, \alpha)-$ good in $V$, then $r f$ is $(C, \alpha)-$ good in $V$ for any $r \in R$.

(b) If $f$ and $g$ are $(C, \alpha)-\operatorname{good}$ in $V$, then $\max \{|f|,|g|\}$ also $(C, \alpha)-\operatorname{good}$ in $V$.

(c) If $f$ is $(C, \alpha)-$ good in $V$, then for any $C^{1} \geq C$, $\alpha^{1} \leq \alpha$ and $V^{1} \subseteq V$, then there is $f$ $\left(C^{1}, \alpha^{1}\right)-\operatorname{good}$ in $V^{1}$.

(d) If $f$ is $(C, \alpha)-$ good in $V$ and $c_{1} \leq \frac{\mid f(x) \|}{\| g(x) \mid} \leq c_{2}$ for all $x \in V$, then $g$ is $\left(C_{y}\left(\frac{C_{2}}{c_{1}}\right)^{\alpha}, \alpha\right)-$ good in $V$. 
To prove: (a) and (b) can be obtained directly from the definition of $(C, \alpha)-$ good function, by changing the $f$ in the definition of $r \times f$ does not affect the results, so $r \times f$ is $(C, \alpha)-\operatorname{good}$. Then $f$ replaced by $\max \{|f|,|g|\}$ does not affect the results, hence, $\max \{|f|,|g|\}$ also $(C, \alpha)-\operatorname{good}$.

The following proves that $(d),(c)$ have detailed proof in the reference [5], because $f$ is $(C, \alpha)-\operatorname{good}$ in $V$, and $c_{1} \leq \frac{\mid f(x) \|}{\| g(x) \mid} \leq c_{2}$, then,

$$
\begin{aligned}
& \mu\left(\left\{x \in B: c_{2} \cdot|g(x)|<\varepsilon \cdot \sup _{x \in B}|f(x)|\right\}\right) \\
& \leq \mu\left(\left\{x \in B:|f(x)|<\varepsilon \cdot \sup _{x \in B}|f(x)|\right\}\right) \\
& \leq C \varepsilon^{\alpha} \mu(B) \\
& \left(\operatorname{so,} \varepsilon=\frac{\varepsilon^{\prime} \cdot \sup |g(x)|}{\sup |f(x)|} \text { then }\right) \\
& =C \cdot\left(\frac{\varepsilon^{\prime} \cdot \sup |g(x)|}{\sup |f(x)|}\right)^{\alpha} \cdot \mu(B) \\
& \leq C \cdot\left(\frac{\varepsilon^{\prime} \cdot \sup |g(x)|}{c_{1} \cdot \sup |g(x)|}\right)^{\alpha} \cdot \mu(B) \\
& =C \cdot\left(\frac{\varepsilon}{c_{1}}\right)^{\alpha} \cdot \mu(B) \\
& \mu\left(\left\{x \in B: c_{2} \cdot|g(x)|<\varepsilon^{\prime} \cdot \sup _{x \in B}|g(x)|\right\}\right) \\
& =\mu\left(\left\{x \in B:|g(x)|<\frac{s^{t}}{c_{2}} \cdot \sup _{x \in B}|g(x)|\right\}\right) \\
& \leq C \cdot\left(\frac{\varepsilon^{\prime}}{c_{1}}\right)^{\alpha} \cdot \mu(B) \\
& \text { (Tomake } \left.\varepsilon^{\mathrm{m}}=\frac{\varepsilon^{\prime}}{c_{2}} \Rightarrow \varepsilon^{\prime}=\varepsilon^{\mathrm{m}} \cdot c_{2} \text { then }\right) \\
& =C \cdot\left(\frac{\varepsilon^{\mathrm{m}} \cdot c_{2}}{c_{1}}\right)^{\alpha} \cdot \mu(B) \\
& =C \cdot\left(\frac{c_{2}}{c_{1}}\right)^{\alpha} \cdot(\varepsilon)^{\alpha \cdot \mu}(B)
\end{aligned}
$$

The next lemma gives a way to judge whether a function is $(C, \alpha)-$ good.

Lemma 2.2: (Reference [5], Lemma 1.3) 
Let $U$ be an open subset of $R^{m}, g \in C^{k}(U)$ makes the constant $A_{1}, A_{2}>0$, for all $x \in U$

$$
\begin{aligned}
& \left|\partial_{\beta} g(x)\right| \leq A_{1}, \forall \beta,|\beta|_{8} \leq k, \\
& \left|\partial_{i}^{k} g(x)\right| \geq A_{2}, \forall i=1, \cdots, m
\end{aligned}
$$

Let $V$ be a subset of $U$ and any external cube of any ball $B$ in $V$ is contained within $U$. So, $g$ is $\left(C, \frac{1}{m k}\right)-g o o d$ on $V$. The constant $C$ depends only on $A_{1}, A_{2}, m, k$.

\section{Proposition 2.1:}

Let $U$ be an open subset of $R^{m}, x_{0} \in U_{3} H \subset C^{(l)}(U)$ is a compact function of $f: U \rightarrow R$, here $l \geq 2$. Suppose

$$
\inf _{f \in H, 0<|\beta|_{*}}\left|\alpha_{\beta} f\left(x_{0}\right)\right|>0
$$

there exist $x_{0}$ domain of $V \subset U$, and the constant numbers $c$ and $\delta$ satisfy the following properties. That is $\forall P \in C^{(l)}(U)$ makes

$$
\sup _{x \in U,|\beta|_{*} \leq l} \max _{\beta}\left|\partial_{\beta} P(x)\right| \leq \delta
$$

and $\forall f \in H$ have

$$
\begin{aligned}
& \text { (a) }: f+P \text { is }\left(C, \frac{1}{m l}\right)-\text { good on } V \\
& \text { (b) }:|\nabla(f+P)| \text { is }\left(C, \frac{1}{m(l-1)}\right)-\text { good. }
\end{aligned}
$$

Proof: Proposition 2.1 is a modification to [6] proposition 1.4.

First of all, there is a constant $C_{1}>0$ in equation(2.4), for any $f \in H$ there is multi index set $\beta$ and $0<|\beta|_{*}=k \leq l$ where $k=k(f)$.

$$
\left|\partial_{\beta} f\left(x_{0}\right)\right| \geq C_{1}
$$

Since the different $\beta$ is limited, without loss of generality suppose that $\beta$ in equation (2.6) satisfies all $f \in H$, through an appropriate coordinate transformation, ensure that for any $i=1,2, \cdots, m$ and constant $C_{2}$ is independent of $f$,

$$
\left|\partial_{i}^{k} f\left(x_{0}\right)\right| \geq C_{2}
$$

where $\tilde{\partial}$ represents the derivative of the rotating coordinate system. From equation (2.5) we can see that there is a constant $c=c(l)>1$ such that

$$
\sup _{x \in U,|\beta| * \leq l}\left|\tilde{\partial}_{\beta} P(x)\right| \leq c \delta
$$

here remember: $\delta=C_{2} / 2 c$ from (4.7) and (4.8) for any $f \in H$ and $i=1,2, \ldots, m$ 


$$
\begin{aligned}
& \left|\tilde{\partial}_{i}^{k}(f+P)\left(x_{0}\right)\right| \\
& \left|\tilde{\partial}_{i}^{k} f(x)+\tilde{\partial}_{i}^{k} P(x)\right| \\
& \geq C_{2}-c \cdot \delta \\
& =C_{2}-c \cdot \frac{C_{2}}{2 c} \\
& \quad=\frac{C_{2}}{2} \\
& \quad \geq \frac{C_{2}}{2 c}=\delta
\end{aligned}
$$

There is continuity of $(f+P)$ and compactness of $H$, recommend $x_{0}$ the field $V^{1} \subset U$, the positive constant number $A_{1}, A_{2}$ independent of $f$, so that $\partial$ can be replaced by $\widetilde{\partial}$. Finally, let $V$ be a smaller field of $x_{0}$ such that the outer cube of any of the balls in $V$ is contained in $V^{1}$, then by lemma 2.2 we can obtain the (a) part of proposition 2.1 .

With respect to (b), it is first assumed that $k$ in equation (4.7) is at least 2, which is compacted by $H$, and its differential is the continuous mapping of the $C^{(l)}(U)$ and $C^{(l-I)}(U)$, for each $i=1, \cdots, m F_{i}:=\left\{\tilde{\partial}_{i} f: f \in H\right\}$, the $C^{(l-I)}(U)$ is compact. By the definition of $H$, we can see that when $l$ is replaced by $l-I, H$ is replaced by $F_{i}$, and conditional (2.4) is also established. Therefore, it is also proved that part (a) is equally applicable to $F_{i}$, and can be applied to each $f \in F_{i}$, function $\widetilde{\partial}_{i}(f+P)$ is $\left(C_{i}, \frac{1}{m(l-l)}\right)-$ good. Here $\widetilde{C}=\max _{i} C_{i}, V=\bigcap_{i} V_{i}, f \in H$.

Naturally, the gradient of the rotational coordinate system is represented by $\widetilde{\nabla}$, notice that $|\nabla(f+P)(x) / \widetilde{\nabla}(f+P)(x)|$ is bounded for all $x \in V$, and between two positive numbers. Thus, the (d) part of lemma 2.1 can be derived from the propositional (b) part. Finally consider when $k=1$ in equation (2.7) let $A_{1}, A_{2}$ and $V$ be defined as in part (a), then for all $x \in V$ have

The definition of the (d) and $(C, \alpha)-$ good functions of Lemma 2.1, we obtain

$$
A_{1} \leq|\widetilde{\nabla}(f+P)(x)| \leq A_{2}
$$

$$
\left|\left\{x \in B:|\widetilde{\nabla}(f(x)+P(x))|<\varepsilon \sup _{y \in B}|\widetilde{\nabla}(f(y)+P(y))|\right\}\right| \leq\left(\frac{A_{1}}{A_{2}}\right)^{\frac{1}{l-I}} \cdot \varepsilon^{\frac{1}{l-I}} \cdot|B|_{m}
$$

for any positive number $\varepsilon$ and any $B \subset V$, if $\varepsilon \geq A_{1} / A_{2}$ then in equation (2.10) the right part is at most $|B|_{m}$, the equation (2.10) clearly established. If $\varepsilon<A_{1} / A_{2}$, the under the condition of equation (2.9), the left side in equation (2.10) is an empty set, equation (2.10) is equally satisfactory.

\section{Corollary 2.1:}

Let $U$ be an open subset of $R^{m}, x_{0} \in U$, let $f=\left(f_{1}, \cdots, f_{n}\right): U \rightarrow R^{n}$ at the point $x_{0}$ is $l$ nondegenerate, for $l \geq 2, P \in C^{(l)}(U)$ there is $x_{0}$ exist in the filed $V \subset U$ and positive constant $c$ and $H_{0}$, for any $q \in R^{n}$ satisfy the $|q| \geq H_{0}$ : 
(a) For each $q_{0} \in R, q_{0}+q \cdot f+P$ is $\left(C, \frac{1}{m l}\right)-$ good in $V$.

(b) $|\nabla(q \times f+P)|$ is $\left(C, \frac{1}{m(l-l)}\right)-$ good in $V$.

Prove: First of all, the domain $V \subset U$ of $x_{0}$ makes $f$ and $P$ bounded on $V$, then there is a constant number $K$ :

$$
\sup _{x \in V}|f(x)| \leq \frac{K}{n+1} \text { and } \sup _{x \in I}|P(x)| \leq \frac{K}{n+1}
$$

Definition: $f(x):=q_{0}+q \times f(x)+P(x)$

Now assume that $\left|q_{0}\right| \geq 2 K|q|$, then by the equation (2.11) can be obtained for an open ball $B \subset V$ $\sup _{x \in B}|f(x)| \leq \underset{x \in B}{\inf _{x \in B}}|f(x)|$ so if $\varepsilon<\frac{1}{3}$, the left side of the equation (2.1) is empty set, this equation satisfies any integer $C$ and $\alpha$. On the other $\varepsilon \geq \frac{1}{3}$, then for any $C$ and positive constant $\alpha \leq 1$, the equation (2.1) is clearly established. When $\left|q_{0}\right| \geq 2 K|\alpha|, \quad \forall C \geq 3$ and $0<\alpha \leq 1$, the corollary (a) is partially true. So when $\left|q_{0}\right| \leq 2 K|q|$, let $H$ be the functions of set $c * f(x)+c_{0}$. Here $c \in R^{n}$ makes $|c|=1,\left|c_{0}\right| \leq 2 K$. Using the compactness of set $\left\{c \in R^{n}:|c|=1\right\} \times\left\{c_{0} \in R^{n}:\left|c_{0}\right| \leq 2 K\right\}$, it can be proved that $H$ is compact in $C^{(l)}(U)$ and $f$ is non-degenerate at $x_{0}$ point, and a satisfying expression is given in equation (2.4). If needed to be reduced the $x_{0}$ into the field $V$, we can get the existence of $M$ which makes sup $\max \left|\partial_{\beta} P(x)\right| \leq M$ (Mis constant), let $C$ and $\partial$ be constant, contact proposition 2.1, let $H_{0}:=M / \delta$ consider any vector $q \in R^{n}$ such that $|q| \geq H_{0}$ and any real number $q_{0}$ and $\left|q_{0}\right| \leq 2 K|q|$ then $\Theta:=P(x) /|q|$ satisfy equation (2.5), that is :

$$
\left|\partial_{\beta} \Theta(x)\right|=\left|\partial_{\beta}\left(\frac{P(x)}{|q|}\right)\right| \leq\left|\partial_{\beta}\left(\frac{P(x)}{H_{0}}\right)\right|=\left|\frac{\delta}{M} \cdot \partial_{\beta} P(x)\right| \leq\left|\frac{\delta}{M}\right| \cdot|M|=|\delta|
$$

and $f: x \rightarrow f(x):=|q|^{-1}\left(q_{0}+f(x) \cdot q+P(x)\right)$ belongs to compact set $H$. By proposition 2.1 function $f+\Theta$ defined as: $f(x)+\Theta(x)=|q|^{-1}\left(q_{0}+f(x) \times q+P(x)\right)$ is the corollary. Finally, we use the lemma 2.1 (a) to remove the multiplication factor $|q|^{-1}$, which leads us to the conclusion.

Proposition 2.2: Let $U, x_{0}$ and $H$ be defined as in proposition 2.1, and assume that (2.4) is valid, then $x_{0}$ in any field $V \subset U$ has $\inf _{f \in H, \sup _{x \in I^{*}}}|f(x)|>0$.

Prove: From (2.4) $\|f\|_{V}:=\sup _{x \in V}|f(x)|>0$ can be obtained for each $f \in H$ and any $x_{0}$ in the filed $V \subset U$, The mapping $f \mapsto\|f\|_{V}$ about the $C^{0}$ norm is continuous, and by the compactness of $H$, we obtain $\inf _{f \in H}\|f\|_{V}=\left\|f_{0}\right\|_{s} f_{0} \in F_{0}$

Corollary 2.2: Let $U_{,} x_{0}, f$ and $P$ as given in corollary 2.1, then for each of the $x_{0}$ a sufficiently small filed of $V \subset U$, there exist $H_{0}>1$ which make $\inf _{\left(q, q_{0}\right) \in R^{n+1},|q| \in H} \sup _{0, x \in V}\left|q_{0}+q \cdot f(x)+P(x)\right|>0$

Prove: Consider $x_{0}$ and any arbitrary filed $V \subset U$ which satisfy the inequality of equation (2.11). 
Let $f(x):=q_{0}+q \times f(x)+P(x)$, then $\left|q_{0}\right| \geq 2 K|q|$, from equation (2.11)

$$
\begin{aligned}
& \sup _{x \in V} \mid f(x) \| \\
& \geq \sup _{x \in V}\left|q_{0}\right|-\sup _{x \in V}|q \cdot f(x)+P(x)| \\
& \geq 2 K|q|-\left(\sup _{x \in V}|q \cdot f(x)|+\sup _{x \in V}|P(x)|\right) \\
& \geq 2 K|q|-\left(|q| \cdot \frac{1}{n+1}+\frac{K}{n+1}\right) \\
& \geq 2 K|q|-\left[|q|\left(\frac{K}{n+1}+\frac{K}{n+1}\right)\right] \\
& \geq 2 K|q|-|q| \cdot\left(\frac{K}{2}+\frac{K}{2}\right)=K|q|^{|q|>H_{0}} \geq K \cdot H_{0}>K>0
\end{aligned}
$$

For any $\left(q, q_{0}\right) \in R^{n+1},|q| \geq H_{0}>1$ and $\left|q_{0}\right| \geq 2 K|q|$. Therefore, the remaining proof assumes $\left|q_{0}\right| \geq 2 K|q|$, and proves corollary 2.1 the same way that $H$ represents a collection of $c * f(x)+c_{0}$, here $c \in R^{n}$ where $|c|=1$ and $\left|c_{0}\right| \leq 2 K$. Then $H$ is the compact subset of $C^{(l)}(U)$, where $f$ is non-degenerate at $x_{0}$ and all $H$ satisfies (2.4). From the proposition 2.2 can see $M:=\inf _{f \in H} \sup |f(x)|>0$ for any

$$
\begin{aligned}
\left(q, q_{0}\right) \in R^{n+1}, \mid q \| \geq H_{0}>1 \text { and }\left|q_{0}\right| \leq 2 K|q| & \\
& \sup _{x \in V}\left|q_{0}+q \cdot f(x)\right| \\
\geq & |q| \cdot\left(\frac{q_{0}}{|q|}+\frac{q}{|q|} \cdot f(x)\right) \\
\geq & |q| \cdot M \\
\geq & H_{0} \cdot M
\end{aligned}
$$

Remember: $H_{0}>\max \{1, K / M\}$.Then form equation (2.11) and equation (2.12) can be obtained

$$
\begin{aligned}
& \sup _{x \in V}\left|q_{0}+q \cdot f(x)+P(x)\right| \\
& \geq \sup _{x \in I^{*}}\left|q_{0}+q \cdot f(x)\right|-\sup _{x \in I^{*}}|P(x)| \\
& \geq M H_{0}-\frac{K}{n+1} \geq M H_{0}-\frac{K}{2} \\
& =M H_{0}-\frac{1}{2} \cdot \frac{K}{M} \cdot M \\
& \geq M H_{0}-\frac{1}{2} M H_{0}=\frac{1}{2} M H_{0}
\end{aligned}
$$


With the help of the non-homogeneous transfer principle, we obtain the following theorem.

Theorem 3.1: Let $M$ be a $l$ non-degenerate submanifold in $R^{n}, P: R^{n} \rightarrow R$ and $\left.P\right|_{M} \in C^{(l)}, \Theta$ is an $n$ element approximation function, when there is $\sum_{q \in Z n \backslash 0\}} \Phi(q)<\infty$ then $\mu\left(A_{n}^{P}(\Phi)\right)=0$ holds.

Where $\mu$ is the $n$-dimensional Lebesgue measure, the Lebesgue measure is non-homogeneous strongly extreme. Where

$A_{n}^{P}(\Phi):=\left\{x \in\right.$ M: there infinitely many $q \in Z^{n} \backslash\{0\}$ such that $\left.\|x q+P(x)\|<\Phi(q)\right\}$.

1.1 Decomposition of sets under convergence

For convenience, make some habitual assumptions here; let smooth mappings $f=\left(f_{1}, \cdots, f_{n}\right): U \rightarrow R^{n}$ also $U \subset R^{m}$. So let $M=\{f(x): x \in U\}$, and the implicit function theorem can be assumed $f_{i}(x)=x_{i}$ here $i=1, \cdots, m$ in other words, $f$ is a parameterized representation of $M$ for Monge, and implies that $f$ is a local biLipschitz. Let $\Omega_{f}(\Phi, P):=\left\{x \in U: f(x) \in A_{n}^{P}(\Phi)\right\}$ represents the projection of $A_{n}^{P}(\Phi)$ on $U$, therefore, when $x \in \Omega_{f}\left(\Phi_{y} P\right)$ if and only if $f(x) \in M$ is $\left(\Phi_{,} P_{f}(x)\right)$ approximation. Here $P_{f}(x):=P(f(x))$, for the convenience of the following: $P_{f}$ when $\Phi(q)=\phi(|q|)$ remember that $\Omega_{f}(\Phi, P)=\Omega_{f}(\phi, P)$. Because the function $f$ and $P$ is $C^{l}$, therefore, without loss of generality, assume the existence of constant $C_{0}>0$ depends only on $U_{s}, P$ which makes

$$
\max _{0 \geq i \geq l} \sup \left|f^{(i)}(x)\right| \leq C_{0} \text { and } \max _{0 \geq i \geq l}\left|P^{(i)}(x)\right| \leq C_{0}
$$

From the above conditions we can see that theorem 1.1

$$
\sum_{q \in Z^{n} \backslash\{0\}} \Phi(q)<\infty
$$

When, we want to get the $\mu\left(\Omega_{f}\left(\Phi_{y} P\right)\right)=0$, the collection $\left(\Omega_{f}\left(\Phi_{j} P\right)\right)$ is written as

$$
\Omega_{f}(\Phi, P)=\lim _{|q| \rightarrow \infty} \sup _{f}(q, \Phi, P):=\bigcap_{h=1}^{\infty} \bigcup_{|q|>h} \Omega_{f}(q, \Phi, P),
$$

Here: $\Omega_{f}(q, \Phi, P):=\{x \in U:\|q * f(x)+P(x)\| \leq \Phi(q)\}$ for each $q \in Z^{n} \backslash\{0\}$ and $A_{f}(q, \Phi, P)$ can be decomposed into

$$
\begin{aligned}
& \Omega_{f}^{1}(q, \Phi, P):=\left\{x \in \Omega(q, \Phi, P):|\nabla(f \cdot q+P(x))(x)| \geq C_{I}|q|^{\frac{1}{2}}\right\} \\
& \Omega_{f}^{2}(q, \Phi, P):=\left\{x \in \mathrm{A}(q, \Phi, P):|\nabla(f \cdot q+P(x))(x)| \geq C_{I}|q|^{\frac{1}{2}}\right\}
\end{aligned}
$$

Where $\nabla$ represents the gradient

$$
C_{I}:=\sqrt{(n+1) m C_{0}}
$$

Obviously $\Omega_{f}(\Phi, P)=\Omega_{f}^{1}(\Phi, P) \cup \Omega_{f}^{2}(\Phi, P)$

Here $\Omega_{f}^{i}(\Phi, P)=\lim _{|q| \rightarrow \infty} \sup \Omega_{f}^{i}(q, \Phi, P):=\bigcap_{h=1}^{\infty} \mathrm{U}_{|q|>h} \Omega_{f}^{i}(q, \Phi, P)(i=1,2)$

To prove that $\mu\left(\Omega_{f}(\Phi, P)\right)=0$ is to prove $\mu\left(\Omega_{f}^{1}(\Phi, P)\right)=0$ and $\mu\left(\Omega_{f}^{2}(\Phi, P)\right)=0$ respectively. Therefore, the points in the manifold discussed can be roughly divided into two categories. In the following articles, the two cases are mainly verified.

\subsection{Proof of theorem}


First, we need to prove the first case $\mu\left(\Omega_{f}^{1}(\Phi, P)\right)=0$, where the theorem in the reference [5] is used, namely this article

Theorem 3.2: (Bernik, Kleinbock and Margulis)

Let $B \in R^{m}$ is a ball with radius $r>0, G=\left(g_{1}, g_{2}, \cdots, g_{n+1}\right) \in C^{(2)}(2 B)$ and assume

$$
L:=\max _{1 \underline{i}_{z} j \underline{m}_{2} x \in 2 B}\left|\frac{\partial^{2} G(x)}{\partial x_{i} \partial x_{j}}\right|<\infty
$$

Then for each $q \in Z^{n+1}$ makes

$$
|q| \geq \frac{1}{4(n+1) L r^{2}}
$$

Satisfying inequality

$$
\left\{\begin{array}{c}
\|G(x) \cdot q\|<\delta \\
\mid \nabla G(x) \cdot q \| \geq((n+1) m L|q|)^{1 / 2}
\end{array}\right.
$$

The measure of the set is at most $K \delta|B|_{m}$ here $x \in B$, and $K$ is a constant that depends only on $m$.

So $B$ is a nonempty ball in $U$ and $2 B \subset U_{3} G=\left(f_{1}, f_{2}, \cdots f_{n}, P\right), q=\left(q_{1}, \cdots, q_{2}, 1\right)$ here $q=\left(q_{1}, \cdots q_{n}\right) \in Z^{n} \backslash\{0\}$, then, in equation (5.1), the above equation (3.5) is satisfied, (3.6) except that a finite number of $q \in Z^{n} \backslash\{0\}$ is established. The inequality of (3.3) is also included in the lower bound inequality of the following equation (3.1) and (3.4).

Thus, when $\delta:=\Phi(q), \Omega_{f}^{1}(q, \Phi, P) \cap B$ is contained in the set defined by equation (3.7). By theorem 3.2 can be obtained, $\mu\left(\Omega_{f}^{1}\left(q, \Phi_{p} P\right) \cap B\right) \ll \Phi(q)$

By equation (3.2) and Borel-Cantell lemma can be deduced immediately $\mu\left(\Omega_{f}^{1}(\Phi, P) \cap B\right)=0$. Obviously meet $2 B \subset U$ the open ball $B$ covers the entire $U$ (the arbitrariness of $B$ ) therefore $\mu\left(\Omega_{f}^{1}(\Phi, P)\right)=0$

To prove a situation, in the proof of this case uses the Good function after deformation and inhomogeneous transfer principle are given in the above, the two categories of content and some related properties mainly lies in the next article in the verification. Our goal is to prove $\mu\left(\Omega_{f}^{2}(\Phi, P)\right)=0$, where $\Phi$ satisfies equations (1.1) and (3.2), and thus available in addition to the finite number of $q$ for all $q \in Z^{n}$

$$
\Phi(q)<\Phi_{0}(q):=\prod_{i=1}^{n}\left|q_{i}\right|^{-1}
$$

Therefore, it is available directly

$$
\Omega_{f}^{2}(\Phi, P) \subset \Omega_{f}^{2}\left(\Phi_{0}, P\right)
$$

As long as the $\mu\left(\Omega_{f}^{2}\left(\Phi_{0}, P\right)\right)=0$ can prove, let $T:=\left(Z_{\geq 0}\right)^{n}$ and $A:=Z^{n} \backslash\{0\} \times Z$

The definition of the auxiliary function

$$
r: T \rightarrow R^{+}, r(t):=\sqrt{2(n+1) m C_{0}} \cdot 2^{\frac{|t|}{2}}
$$


Here $C_{0}$ is given in equation (3.1), then $\varepsilon>0$ is given, $t \in T$ and $\alpha=\left(q, q_{0}\right) \in A$ Let

$$
I_{t}(\alpha, \varepsilon):=\left\{\begin{array}{c}
\left|q_{0}+q \cdot f(x)+P(x)\right|<\varepsilon \cdot \Phi_{0}\left(2^{t}\right) \\
x \in U:|\nabla(q \cdot f(x)+P(x))|<\varepsilon \cdot r(t) \\
2^{t_{i}} \leq \max \left\{1,\left|q_{i}\right|\right\}<2^{t_{i}+1}(1 \leq i \leq n)
\end{array}\right.
$$

And

$$
H_{f}(\alpha, \varepsilon):=\left\{\begin{array}{c}
\left\|q_{0}+q \cdot f(x)\right\|<2 \varepsilon \cdot \Phi_{0}\left(2^{t}\right) \\
x \in U:|\nabla(q \cdot f(x))|<2 \varepsilon \cdot r(t) \\
\left\|q_{i}\right\|<2^{t_{i}+2}(1 \leq i \leq n)
\end{array}\right.
$$

Here $2^{t}:=\left(2^{t_{n}} \ldots, 2^{t_{n}}\right), H$ and $I$ are define in equation (1.2), also given $\delta \in R$

$$
\psi_{\delta}:=T \rightarrow R^{+}, \psi_{\delta}(t):=2^{\delta|t|}
$$

Let $\Psi:=\left\{\psi_{\delta}: 0 \leq \delta \leq \frac{1}{4}\right\}$ for any $\delta \in\left[0, \frac{1}{4}\right)$ can get $\Omega_{f}^{2}(\Phi, P) \subset \Lambda_{I}\left(\psi_{\delta}\right)$ where $\Lambda_{I}\left(\psi_{\delta}\right)$ define as in (1.3), therefore, the second case can be established by (3.9), that is to prove $\delta \in[0,1 / 4)$

$$
\mu\left(\Lambda_{I}\left(\psi_{\delta}\right)\right)=0
$$

Here, $x_{0}$ is any point in $U, f$ is the $l$-non-degenerate, $x_{0}$ the small ball in $V$.

Theorem 3.3: (Reference [5] Theorem 2.1)

Let $x_{0} \in U_{3} f: U \rightarrow R^{n}$ at point $x_{0}$ is $l$ - non-degenerate, then there is $x_{0}$ in the field $V \subset U$ have the following properties. For the arbitrary ball $B \subset V$ there exist $E>0$ so that the optional real number $\omega, K_{,} T_{1}, \cdots, T_{n}$ satisfies the following inequality $0<\omega<1$,

$$
\begin{array}{r}
T_{1}, \cdots, T_{n} \geq 1, K>0 \text { and } \frac{\omega K T_{1} \cdots T_{n}}{\max T_{i}} \leq 1 \\
S\left(\omega, K, T_{1}, \cdots, T_{n}\right):=\left\{\begin{array}{c}
\|f(x) \cdot q\|<\omega \\
x \in B: q \in Z^{n}|\nabla f(x) \cdot q|<k \\
\left|q_{i}\right|<T_{i}(1 \leq i \leq n)
\end{array}\right\}
\end{array}
$$

The $m$ dimensional Lebesgue measure is at most $E \times \varepsilon^{\frac{1}{m(2 I-1)}}=|B|_{m}$ here

$$
\varepsilon:=\max \left(\omega,\left(\omega k T_{1} \cdots T_{n} / \max T_{i}\right)^{1 / n}\right)
$$

Let $\mu$ be a Lebesgue measure for $m$ on $V$, and $f$ is $l$-non-degenerate to prove that equation (3.14) which proof

$$
\exists \delta \in\left[0, \frac{1}{4}\right) \text { has } \mu\left(\Lambda_{I}\left(\psi_{\delta}\right)\right)=0
$$

Using the non-homogeneous transfer principle, it is further assumed that $(H, I, \Psi)$ satisfies the intersection property and $\mu$ about $(I, \Psi)$ satisfies the contraction properties. In theorem 3.3 the construction of equation (3.16) is proof

$$
\exists \delta \in\left[0, \frac{1}{4}\right) \operatorname{has} \mu\left(\Lambda_{H}\left(\psi_{\delta}\right)\right)=0
$$


Fixed $\forall \delta \in\left[0, \frac{1}{4}\right)$ by equation (3.12)

$$
\bigcup_{\alpha \in A} H_{t}\left(\alpha, \psi_{\delta}(t)\right)=S\left(\omega, K, T_{1} \cdots T_{n}\right)
$$

Let $\omega=2 \psi_{\delta}(t) \cdot \Phi_{0}\left(2^{t}\right)$ also $K=2 \psi_{\delta}(t) \cdot r(t)$ and $T_{i}=2^{t_{i}+2}$ here $(1 \leq i \leq n)$ by theorem 3.3 and equation (3.8), the exact value of $\Phi_{0}\left(2^{t}\right), r(t)$ and $\psi_{\delta}(t)$ is given

Form equation (5.15) defines $\varepsilon$ which satisfying $\varepsilon \leq 2^{\frac{\left(\frac{1}{2}-n \delta\right)}{n+1}\|t\|}$ by theorem 3.3

We can get

$$
\mu\left(\bigcup_{\alpha \in A} H_{t}\left(\alpha, \psi_{\delta}(t)\right)\right) \ll 2^{-r \mid t]}
$$

Where $r:=\frac{\left(\frac{1}{2}-2 \delta\right)}{m(n+1)(2 l-1)}$ is a positive number,

$$
\sum_{t \in T} \mu\left(\bigcup_{\alpha \in A} H_{t}\left(\alpha, \psi_{s}(t)\right)\right) \ll \sum_{t \in Z^{n}} 2^{-r|t|}<\infty
$$

Finally, the Borel-Cantelli lemme can be obtained $\mu\left(\Lambda_{H}\left(\psi_{\delta}\right)\right)=0$

It is necessary to check the rationality of the non-homogeneous transfer principle under the conditions of this paper, that is, only the intersection condition and the contraction condition are verified.

First, check the intersection condition:

Let $t \in T$ and $|t| \geq 2$ assume that $x \in I_{t}\left(\alpha, \psi_{\delta}(t)\right) \cap I_{t}\left(\alpha^{\prime}, \psi_{\delta}(t)\right)$ is different for $\alpha=\left(q, q_{0}\right)$ and $\alpha^{\prime}=\left(q^{g}, q_{0}^{y}\right)$ then equation (3.11) and (3.12) can be obtained

$$
\begin{aligned}
& \left\{\begin{array}{l}
\left|q_{0}+q \cdot f(x)+P(x)\right|<\psi_{s}(t) \cdot \Phi_{0}\left(2^{t}\right) \\
\left|q_{0}^{s}+q^{s} \cdot f(x)+P(x)\right|<\psi_{s}(t) \cdot \Phi_{0}\left(2^{t}\right)
\end{array}\right. \\
& \left\{\begin{array}{l}
|\nabla(q \cdot f(x)+P(x))|<\psi_{s}(t) \cdot r(t) \\
\left|\nabla\left(q^{s} \cdot f(x)+P(x)\right)\right|<\psi_{s}(t) \cdot r(t)
\end{array}\right. \\
& \left\{\begin{array}{l}
\left|q_{i}\right|<2^{t_{i}+1} \\
\left|q_{i}^{s}\right|<2^{t_{i}+1}
\end{array},(1 \leq i \leq n)\right.
\end{aligned}
$$

Where $\left(q_{1}, \cdots, q_{n}\right)=q$ and $\left(q_{1}^{y}, \cdots, q_{n}^{y}\right)=q^{y}$ subtract the second equation from the first equation, respectively

$$
\left\{\begin{array}{c}
\left|q_{0}{ }^{\mathrm{I}}+q^{\mathrm{m}} \cdot f(x)\right|<2 \psi_{\delta}(t) \cdot \Phi_{0}\left(2^{t}\right) \\
\left|\nabla\left(q^{\mathrm{m}} \cdot f(x)\right)\right|<2 \psi_{\delta}(t) \cdot r(t) \\
\left|q_{i}^{\mathrm{m}}\right|<2^{\mathrm{t}_{\mathrm{i}}+2}, 1 \leq i \leq n
\end{array}\right.
$$

The first inequality of the above equation is given by (3.8) and $\Psi$ and are defined as:

$$
\psi_{\delta}(t) \cdot \Phi_{0}\left(2^{t}\right)=2^{\delta|t|} \cdot \prod_{i=1}^{n}\left|2^{t_{i}}\right|^{-1}<2^{\delta|t|} \cdot 2^{-|t|^{\delta=1 / 4}}<2^{\left.-\frac{3}{4}|t|\right]}
$$

Suppose $q^{m}=0$ at the moment, because $\alpha, \alpha^{\prime} \in A$ is different, there must be $q_{0}^{m} \neq q_{0}$ called $\left|q_{0}^{m}+q^{m} \cdot f(x)\right|=\left|q_{0}^{m}\right| \geq 1$ however, $t$ for any $|t| \geq 2$, this negates the first inequality of (3.18) so that $q^{m} \neq 0$ for all $\alpha^{m} \in A, x \in H_{t}\left(\alpha^{m}, \psi_{\delta}(t)\right)$ finally makes $\psi^{*}=\psi_{\delta}$ which satisfies the equation (1.4).

Then check the contraction property: 
So that $V$ is a sufficiently small open ball, so that inference 2.1 valid on $5 \mathrm{~V}$, so there are positive numbers $H_{0}$ and $C$ such that for any $t \in T$ and $\alpha=\left(q, q_{0}\right) \in A$ satisfy|q| $\geq H_{0}$, and $q_{0}+q \times f(x)+P(x)$ and $|\nabla(q \circ f(x)+P(x))|$ are $\left(C, \frac{1}{m l}\right)-$ good in $5 V$. By lemma 2.1, the arbitrary $t \in T$ and $\alpha=\left(q, q_{0}\right) \in A$ that is $|q| \geq H_{0}$,

$$
\text { There is } F_{t_{i} \alpha} \text { in } 5 V \text { is }\left(C, \frac{1}{m l}\right)-\operatorname{good}
$$

Here $F_{t, \alpha}: U \rightarrow R, F_{t_{\alpha} \alpha}:=\max \left\{\Phi_{0}^{-1}\left(2^{t}\right) \times r(t)\left|q_{0}+q \times f(x)+P(x)\right|,|\nabla(q \times f(x)+P(x))|\right\}$ the first two inequalities of equation (3.11) are equivalent to $F_{t, \alpha}(x)<\varepsilon \times r(t)$. Thus by definition

$$
\begin{aligned}
& I_{t}(\alpha, \varepsilon)=\left\{x \in U: F_{t, \alpha}(x)<\operatorname{cr}(t)\right\} \\
& 2^{t_{i}} \leq \max \left\{1,\left\|q_{i}\right\|<2^{t_{i}+1}, \quad(1 \leq i \leq n)\right.
\end{aligned}
$$

It is clear that when equation (3.21) is not established $I_{t}(\alpha, \varepsilon)$ is an empty set independent of $\varepsilon$. This $\psi_{\delta} \in \Psi$ makes $\psi_{\delta}^{+}:=\psi_{\frac{1}{2}}\left(\delta+\frac{1}{4}\right)$ available for all $t \in T$ and $\psi_{\delta}(t) \leq \psi_{\delta}^{+}(t)$. Therefore

$$
I_{t}\left(\alpha, \psi_{\delta}(t)\right) \subset I_{t}\left(\alpha, \psi_{\delta}^{+}(t)\right)
$$

Now construct the $V$ in the set $C_{I_{u} \alpha}$, a suitable column of $k_{t}$ satisfies the conditional expression (1.6) to (1.8). If $I_{t}\left(\alpha, \psi_{\delta}(t)\right)$ is an empty set, it is clear that $C_{t_{i} \alpha}$ is an empty set, so suppose that equation (3.21) is true and $I_{t}(\alpha, \varepsilon)$ is given by equation (3.20), (3.8) and the definition of $\Psi$ can be obtained:

$I_{t}\left(\alpha, \psi_{\delta}^{*}(t)\right) \subset\left\{x \in U:\left|q_{0}+q \cdot f(x)+P(x)\right|<2^{-\frac{\pi}{4}|t|}\right\}$ has pointed out that, $q_{0}+q \cdot f(x)+P(x)$ on the $5 \mathrm{~V}$ is $\left(C, \frac{1}{m l}\right)-$ good for all sufficiently large $|q|$. Thus, by definition of $(C, \alpha)-$ good and inference of $2.2|t|$ is sufficiently large

$$
\mu\left(\left(I_{t}\left(\alpha, \psi_{s}^{+}(t) \cap V\right)\right)\right) \leq\left(\left\{x \in V:\left|q_{0}+q \cdot f(x)+P(x)\right|<2^{\left.-\frac{3}{4}|t| t\right]}\right\}\right) \ll 2^{-\frac{3] t \mid t]}{4 m l} \cdot \mu(V) .}
$$

So for all sufficiently large $|t|$,

$$
I_{t}\left(\alpha, \psi_{\delta}^{+}\right) \notin V
$$

From (3.22) and the fact is that $I_{t}\left(\alpha, \psi_{\delta}^{+}\right)$is open set, for each $x \in S \cap I_{t}\left(\alpha, \psi_{\delta}(t)\right)$ there is a $x$ that is $B^{\prime}(x)$ of the ball

$$
B^{\prime}(x) \subset I_{\delta}\left(\alpha, \psi_{\delta}^{+}(t)\right)
$$

And the combination of (3.23), (3.24) and $V$ are bounded, then there is a scaling factor $\tau \geq 1$ that makes the ball $B(x):=\tau B^{\prime}(x)$ satisfied

$$
\begin{gathered}
S \cap B(x) \subset I_{t}\left(\alpha, \psi_{\delta}^{+}(t)\right) \\
S \cap 5 B(x) \not I_{t}\left(\alpha, \psi_{\delta}^{+}(t)\right) \\
5 B(x) \subset 5 V
\end{gathered}
$$


Now that $C_{I_{0} \alpha}:=\left\{B(x): x \in S \cap I_{t}\left(\alpha, \psi_{\delta}(T)\right)\right\}$ be constructed from the above (3.25), the conditional expressions (1.6) and (1.7) are automatically satisfied. With respect to (1.8) consider the any $B$ in $C_{t, \alpha}$, from (3.20) and (3.25) the following expression can be obtained:

$$
\begin{gathered}
\sup _{x \in 5 B} F_{t_{j} a}(x) \geq \sup _{x \in 5 B \cap S} F_{t_{j} a}(x) \geq \psi_{\delta}^{+}(t) \cdot r(t) \\
\sup _{x \in 5 B \cap_{I t}\left(\alpha, \psi_{\delta}(t)\right)} F_{t_{j} a}(x) \leq \psi_{\delta}(t) \cdot r(t)
\end{gathered}
$$

Then by definition of $\psi_{\delta}, \psi_{\delta}^{+}, r(t)$ and expression of (3.27) and (3.28) we can see

$$
\sup _{x \in 5 B \cap I t\left(\alpha, \psi_{\delta}(t)\right)} F_{\mathrm{t}_{j} \alpha}(x) \leq 2^{-\frac{1}{2}\left(\frac{1}{4}-\delta\right)|t|} \cdot \sup _{x \in 5 B} F_{\mathrm{t}_{t} \alpha}(x)
$$

When expression (3.21) is established, there is $|\alpha|>H_{0}$ for sufficiently large $|t|$, therefore, expression (3.19) is valid, and (3.26) and (3.29) can be obtained.

$$
\begin{aligned}
& \mu\left(5 B \cap I_{t}\left(\alpha, \psi_{\delta}(t)\right)\right) \\
\leq & \mu\left(\left\{x \in 5 B:\left|F_{t_{i} \alpha}(x)\right| \leq 2^{-\frac{1}{2}\left(\frac{1}{4}-\delta\right)|t|} \cdot \sup _{x \in 5 B} F_{t_{i} \alpha}(x)\right\}\right) \\
\leq & C \cdot 2^{-\delta^{*}|t|} \cdot \mu(5 B)
\end{aligned}
$$

Here: $\delta^{*}:=\frac{1}{2}\left(\frac{1}{4}-\delta\right) \frac{1}{m l}>0$ therefore, from (3.30) means that there is a limited number of $t \in T$ besides

$$
\mu\left(5 B \cap I_{t}\left(\alpha, \psi_{s}(t)\right)\right) \leq C \cdot 2^{\left.-6^{*} \mid t\right]} \cdot \mu(5 B)
$$

Here: $k_{t}:=C \cdot 2^{-\delta^{*}|t|}$ this verifies the condition of the contraction property (1.8), and the convergence condition $\sum_{t \in T} k_{t}<\infty$ is satisfied. The proof of the above is to satisfy the two conditions of the non-homogeneous conversion principle, therefore, satisfying the non-homogeneous transformation theorem. Thus the theorem $\mathbf{3 . 1}$ is proved.

\section{Conclusion}

Diophantine approximation is one of the important branch of the number, from the most basic rational approximation to Diophantine manifold is now very active on approximation is attracted more and more attention in recent years, also made some important achievements. In this paper, we mainly discuss the non-homogeneous convergence theorem of Diophantine approximation restricted to submanifold $M$ on $R^{n}$, on the basis of the development of the Barker-Sprindzuk conjecture and the non-homogeneous approximation theory and the development of the homogeneous Groshev type Diophantine approximation theory, by classifying the midpoint of the manifold discussed in this paper, we use the Borel Cantell lemma and (C, a) - good lemma and its properties as well as the most important non-homogeneous transformation principle, in this paper, we discuss the Lebesgue measure of these two sets in the case of series convergence, and give the measure theory of Groshev type non-homogeneous Diophantine approximation, that is the Lebesgue measure is nonhomogeneous strongly extreme. In this paper, the non-homogeneous strong extremism of a measure with parametric matrix is further generalized and given by the fact that the friendly measure is a strong contraction.

This paper only discusses the Lebesgue measure of the set in the case of convergence of the series, but the measure in the divergence case has not been completely solved; and the Hausdorff measure in the convergence of the series also needs to be considered; combining the non-homogeneous theory of the Diophantine approximation under the complete divergence given in [4], whether some of the existing results under homogeneous conditions can be extended to nonhomogeneous cases, and whether the monotonicity of the approximation functions in the discussed theorems can be eliminated, these issues remain to be discussed later.

\section{REFERENCES}

[1] Beresnevich V, Velani S. An inhomogeneous transference principle and Diophantine approximation[J]. Proceedings of the London Mathematical Society, 2010, 101(3), 821-851

[2] Bernik V, Kleinbock D, Margulis G A. Khintchine-type theorems on manifolds: the convergence case for standard and multiplicative versions[J]. International Mathematics Research Notices. 2001, 9: 453-486 
[3] D.Badziahin, V.Beresenvich and S.Velani. Inhomogeneous theory of dual Diophantine approximation on manifolds[J]. AMS Subject classification. 2013, 11J83, 11J13, 11J60

[4] D.Kleinbock and .A.Margulis. Flows on homogeneous spaces and Diophantine approximation on manifolds[J]. Ann. of Math. 1998, 148: $339-360$

[5] V.Bernik, D.Kleinbock and G.A.Margulis. Khinctine-type theorems on manifolds: the convergence case for standard and multiplicative version[J]. Internat. Math. Res. Notices. 2001, 9: 453-486

[6] Tapas Kumar Chandra. The Borel-Cantelli Lemma[J]. India, Springer Briefs in Statistics. 2013, 2: 15-18

\section{Author' biography with Photo}

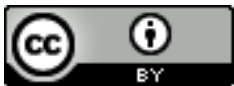

This work is licensed under a Creative Commons Attribution 4.0 International License. 